\title{
Primordial black holes from Higgs vacuum instability: avoiding fine-tuning through an ultraviolet safe mechanism
}

\author{
J. R. Espinosa ${ }^{1,2, a}$, D. Racco $^{3, b}\left(D\right.$, A. Riotto $^{3, c}$ \\ ${ }^{1}$ Institut de Física d'Altes Energies (IFAE), The Barcelona Institute of Science and Technology (BIST), Campus UAB, Bellaterra, 08193 Barcelona, \\ Spain \\ 2 ICREA, Institució Catalana de Recerca i Estudis Avançats, Passeig de Lluís Companys 23, 08010 Barcelona, Spain \\ ${ }^{3}$ Département de Physique Théorique and Centre for Astroparticle Physics (CAP), Université de Genève, 24 quai E. Ansermet, 1211 Geneva, \\ Switzerland
}

Received: 2 May 2018 / Accepted: 22 September 2018 / Published online: 4 October 2018

(C) The Author(s) 2018

\begin{abstract}
We have recently proposed the idea that dark matter in our universe is formed by primordial black holes generated by Standard Model Higgs fluctuations during inflation and thanks to the fact that the Standard Model Higgs potential develops an instability at a scale of the order of $10^{11} \mathrm{GeV}$. In this sense, dark matter does not need any physics beyond the Standard Model, although the mechanism needs fine-tuning to avoid the overshooting of the Higgs into the dangerous AdS vacuum. We show how such finetuning can be naturally avoided by coupling the Higgs to a very heavy scalar with mass $\gg 10^{11} \mathrm{GeV}$ that stabilises the potential in the deep ultraviolet, but preserving the basic feature of the mechanism which is built within the Standard Model.
\end{abstract}

\section{Introduction}

In a recent paper [1] we have proposed the idea that the Standard Model (SM) of weak interactions might provide a candidate for dark matter, without the need of physics beyond the SM, under the form of Primordial Black Holes (PBHs). The scenario is the following. As is well-known, the Higgs potential develops an instability at large values of the field [2-13]. For instance, the Higgs potential becomes negative for Higgs field values of the order of $10^{11} \mathrm{GeV}$ if we select the present central values of the Higgs and top masses. At this scale, the quartic coupling $\lambda$ in the Higgs potential turns negative. During inflation, which is necessary to explain the anisotropies in the cosmic microwave background radiation as well as to provide the seeds for the large-scale structure, the

\footnotetext{
a e-mail: espinosa@ifae.es

b e-mail: davide.racco@unige.ch

c e-mail: antonio.riotto@unige.ch
}

Higgs field is subject to quantum fluctuations as any other field lighter than the Hubble rate $H$ [14]. The Higgs field performs a random walk which allows it to surmount the barrier of its effective potential.

After surmounting the barrier, the Higgs continues its random walk till it reaches the field value at which the classical motion dominates over the quantum jumps. This happens when $\Delta_{c} h \simeq-V^{\prime}(h) / 3 H \gtrsim(H / 2 \pi)$, or

$h^{3} \gtrsim \frac{3 H^{3}}{2 \pi \lambda}$,

where for simplicity we have approximated the Higgs potential as $V(h)=-\lambda h^{4} / 4$. The Higgs field starts a slow-roll phase till it reaches the value $h^{2} \sim 3 H^{2} / \lambda$ at which slow-roll ends and the Higgs starts moving rapidly towards the bottom of the potential. The would-be inevitable destiny of the Higgs field to create a crunching anti de Sitter region is however changed by the fact that inflation ends, the universe reheats at a temperature $T_{\mathrm{RH}}$ and the plasma particles generated during reheating couple to the Higgs, providing to its effective potential a large positive mass that can stop the fall of the Higgs field. Afterwards, the Higgs starts oscillating around our safe electroweak vacuum and promptly decays. Meanwhile, Higgs quantum perturbations are generated. During inflation they leave the Hubble radius and the corresponding curvature perturbations freeze in, while the total curvature perturbation grows [1]. The Higgs contributes to the curvature perturbation with a peak at small scales, when there are about 20 e-folds to go till the end of inflation. During the radiation phase that immediately follows the end of inflation, the Higgs decays communicating its perturbations to the curvature perturbation now in the form of radiation. The final curvature perturbation is therefore flat on large scales, but has a peak at small scales. When these small-scale wavelengths re-enter the horizon, if large enough, the may origi- 
nate PBHs with a mass roughly equal to the mass contained in that horizon volume and with a mass fraction

$\beta_{\text {prim }} \simeq \frac{\Delta_{c}}{\sqrt{2 \pi} \sigma_{\Delta}} e^{-\Delta_{c}^{2} / 2 \sigma_{\Delta}^{2}}$,

where

$\Delta(\mathbf{x})=\frac{4}{9 a^{2} H^{2}} \nabla^{2} \zeta(\mathbf{x})$,

is the density contrast (during the radiation era), $a$ is the scale factor, $\Delta_{c}$ is the critical value above which a given region collapses to a PBH (its value is typically $\sim 0.45$ ), $\zeta$ is the gauge-invariant curvature perturbation, and we have defined the variance of the density contrast to be

$\sigma_{\Delta}^{2}(M)=\int \mathrm{d} \ln k W^{2}\left(k, R_{H}\right) \mathscr{P}_{\Delta}$.

Here $W^{2}\left(k, R_{H}\right)$ is a Gaussian window function smoothing out the density contrast on the comoving horizon length $R_{H}=1 / a H$. The present dark matter abundance made of PBHs of mass $M$ is therefore given by

$$
\left(\frac{\Omega_{\mathrm{DM}}(M)}{0.12}\right) \simeq\left(\frac{\beta_{\text {prim }}(M)}{7 \cdot 10^{-9}}\right)\left(\frac{106.75}{g_{*}}\right)^{1 / 4}\left(\frac{M_{\odot}}{M}\right)^{1 / 2},
$$

where $g_{*}$ is the effective number of degrees of freedom at the time of PBH formation. In this expression we have neglected accretion which would eventually allow to start from smaller PBH mass fractions at formation. The results of Ref. [1] have been repeated, confirmed and reported in Ref. [15].

\section{The fine-tuning}

As any other model of inflation which creates PBHs out of spiked perturbations at small scales, the scenario we have described is fine-tuned $[1,15]$. Indeed, the mass fraction (2) is exponentially sensitive to the variance of the density contrast. Small variations of it lead to too small or too large PBH abundances. In Ref. [1] the fine-tuned choice of the parameters has been motivated anthropically. Structures can form through the dark matter under the form of PBHs and life can develop only in those regions which survive the AdS catastrophe and are saved by the thermal effects. In this sense, the electroweak SM instability is a bonus.

Let us elaborate on fine-tuning. The abundance of PBHs is sensitive to the initial condition of the Higgs field $h_{*}$ at the time when classicality takes over, that is $\sim 20$ e-folds before the end of inflation. Small deviations from $h_{*}, \delta h_{*} \simeq\left(10^{-3}-\right.$ $\left.10^{-2}\right) H$ lead to a too tiny value of the PBH abundance or to a fall into anti de Sitter. This variation has to be compared to the quantum fluctuations $\pm(H / 2 \pi) \simeq 0.16 H$, which arise on length scales $\sim H^{-1}$ during the first $e$-folds of evolution of the Higgs field. This gives a small probability $\sim 10^{-2}$ for each Hubble volume that the initial condition stays close to $h_{*}$.

Since our observed universe (corresponding to a number of e-folds of about 60) contained at that time about $\exp (120)$ Hubble volumes, one might naively think that the total prob-

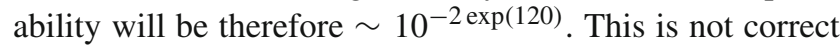
as one is not interested in the probability of simultaneous production of PBHs in all Hubble size domains. The probability though applies to the counting of regions with might end up not being saved by the thermal effects. If one of those $\sim \exp (120)$ regions is not saved, it will expand after inflation and eventually engulf our entire universe. For each of these regions, it was argued that the probability for the Higgs to be saved is of order $1 / 2$ [15]. The reason is that, in the regions that give an abundance of PBHs of the order of $\Omega_{\mathrm{DM}}$, the Higgs field reaches a final value $h_{\mathrm{c}}\left(t_{\mathrm{e}}\right)$ very close to the critical value not to create a dangerous AdS bubble. A small overfluctuation of $h_{*}$ or its initial velocity $\dot{h}\left(t_{*}\right)$ would push then $h_{\mathrm{c}}\left(t_{\mathrm{e}}\right)$ into the AdS regime. Thus, in Ref. [15] it is argued that the probability that none of the $\sim \exp (120)$ regions makes an AdS bubble is $2^{-\exp (120)}$.

In the published PRL version of our paper, now v2 of Ref. [1], it was already explicitly stated that the choice of parameters needed for $\mathrm{PBH}$ formation, although finetuned, would be motivated anthropically. The relevant issue is then the following: is $\sim 2^{-\operatorname{exp(120)}}$ really a small number from the point of view of the multiverse and anthropic argument? In fact, once one accepts the anthropic principle, the reasonable question is what one should multiply the tiny probability for? Within the eternal inflation/multiverse, one should use the volume-weighted physical probability which, unlike the comoving probability distribution, takes into account the overall growth of the volume of the universe: inflationary growth rewards parts of the universe with respect to others. If one assumes a comoving probability point of view, a sample is assumed to be typical and then general properties are deduced. However, distributions looking atypical from an analysis based on the comoving probability, can be common when using the physical probability (and vice versa). We might well live in a region of the global universe which looks unusual if judged so using the comoving probability [16]. In other words, there might be a number of universes much bigger than $\sim 2^{\exp (120)}$ to probe.

As an example, we can refer to Ref. [17] where it is estimated that the number of universes in eternal inflation is proportional to the exponent of the entropy of inflationary perturbations, $\exp (\exp (3 N))$, where $N$ is the number of efolds of slow-roll post-eternal inflation. If we assume that our observed universe originates from only $60 \mathrm{e}$-folds of exponential expansion, one gets [17]

$\mathscr{N}=$ number of universes $\sim 10^{10^{77}}$. 
This is incomparably larger than the number $\mathscr{N}$ of universes needed to find, with a probability of order one, $n=\exp (120)$ adjacent regions which have not fallen into AdS. This probability can be approximated as $2^{-n} \mathscr{N}$, which gives $\mathscr{N} \gtrsim$ $2^{\exp (120)} \sim 10^{4 \cdot 10^{51}}$. In chaotic inflation where the number of e-folds is typically $10^{12}$ one gets [17]

$\mathscr{N} \sim 10^{10^{10^{7}}}$

As scary as it might seem, the small probability quoted in Ref. [15] takes an (exponentially) enormous advantage of this number of universes and what seems unnatural in fact might turn out to be natural. For instance, as shown in Ref. [18], bubbles of AdS may shrink during inflation, if they start with small radius and low velocity. Suppose that half of them shrink and half of them expand. This implies again that one just needs to live in that patch among the exponentially large number where all of the dangerous bubbles shrink and this probability in the multiverse will not be exponentially small, but order unity.

Furthermore, if one wishes to estimate the probability of survival of our universe, it should also be remembered that luckily we live again in a period when the cosmological constant dominates. It can be easily calculated that the particle horizon in our universe from now until infinity will expand by just one third with respect to its current value. From that moment on, our universe will be screened against AdS bubbles.

Leaving aside these considerations which might render the reader (and us) uncomfortable for the lack of any firm quantitative arguments, in this paper we will propose a natural solution to the fine-tuning problem. As mentioned above, the fine-tuning caused demanding the right abundance of PBHs is only $\sim\left(10^{-3}-10^{-2}\right)$. The problem arises when discussing the fine-tuning needed to save all the Hubble volumes when there are about 20 e-folds to go. So, one just needs a (reasonable) solution which will eliminate the presence of the AdS regions altogether without altering the attractive properties of the scenario, i.e. that the PBHs are generated by the SM Higgs and that dark matter is made of SM particles. This is what we will discuss in the next section.

\section{Getting rid of the AdS regions altogether}

As we stressed in Ref. [1] the mechanism to produce PBHs, which today form the dark matter without resorting to any dark matter particle beyond the Standard Model, relies on the instability of the electroweak vacuum, so that the Higgs perturbations can grow during inflation. This dynamics is totally built within the Standard Model. On the other hand, to get rid of the fine-tuning caused by the dangerous AdS vacua, one can simply alter the form of the Higgs potential at

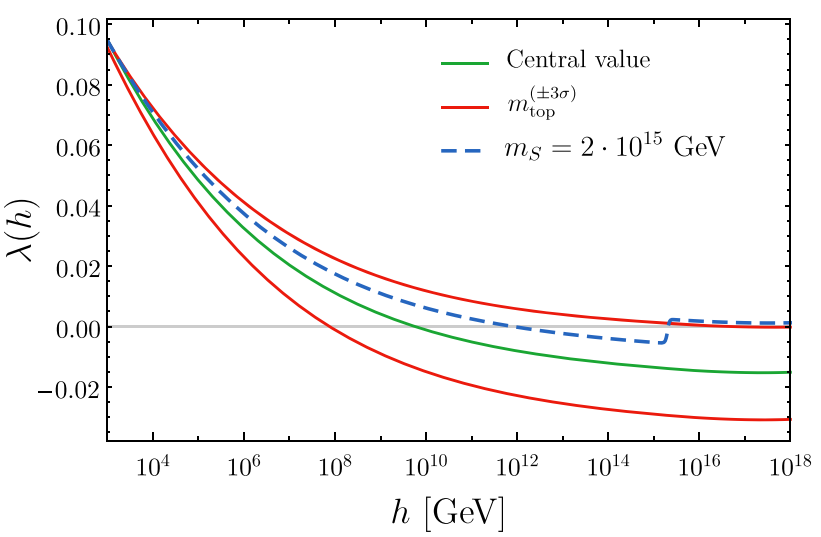

Fig. 1 Running of the Higgs quartic coupling $\lambda$ with the introduction of an extra scalar (dashed blue line), together with the central and marginal values within the SM [11-13]

energies much larger than the instability scale, thus without altering the nice features of the mechanism.

Let us then suppose that at energy scales much larger than the instability scale $\sim 10^{11} \mathrm{GeV}$, there are new particles whose interaction with the Higgs can change the sign of the quartic coupling from negative to positive again, thus stabilising the Higgs potential. As a simple case, add a complex scalar field $S$ with potential [19]

$V=\lambda_{S}\left(|S|^{2}-\omega^{2} / 2\right)^{2}+2 \lambda_{H S}\left(|S|^{2}-\omega^{2} / 2\right)\left(\left|\varphi_{H}\right|^{2}-v^{2} / 2\right)$,

where $S$ is the additional singlet (with vacuum expectation $\omega)$ and $\varphi_{H}$ the SM Higgs doublet (with vacuum expectation $v$ ). The interaction term in Eq. (8), generates a threshold contribution $\delta \lambda=-\lambda_{H S}^{2} / \lambda_{S}$ to the Higgs quartic $\lambda(h)$ above a scale $\sim m_{S}$ [19]. Moderate values of $\lambda_{H S}$ and $\lambda_{S}$ can produce a $\delta \lambda$ large enough to bring $\lambda(h)$ to be positive for $h \gtrsim m_{S}=\sqrt{\lambda_{S}} \omega$. In such case the Higgs potential displays a true minimum at $h \sim m_{S}$.

For our purposes we consider the case $m_{S} \sim \mathscr{O}\left(T_{\mathrm{RH}}\right)$. In this way, even if the Higgs jumps beyond the barrier early during inflation, it will stop at its true minimum and thermal effects at reheating rescue the Higgs, bringing it back towards the electroweak vacuum.

We show in Fig. 1 the running of $\lambda$ corresponding to $m_{S}=$ $2 \cdot 10^{15} \mathrm{GeV}$, and $\lambda_{S H}=0.05, \lambda_{S}=0.3$. We stress that there is no fine-tuning here: we can allow relative variations of order $(10-20) \%$ for $m_{S}$, and the only requirement for $\lambda_{S H}$ and $\lambda_{S}$ is that they yield $\lambda\left(h \gtrsim m_{S}\right)>0$, corresponding to $|\delta \lambda| \gtrsim 0.008$.

We repeat the analysis in Ref. [1], with the same choice of the Hubble rate $H=10^{12} \mathrm{GeV}$ and $m_{\text {top }}=172 \mathrm{GeV}$. The Higgs field starts its classical evolution beyond the barrier at $10^{11} \mathrm{GeV}$ from a value $h_{*}$ at the time $t_{*}$ (corresponding to $N_{*} e$-folds till the end of inflation). We solve the equations 


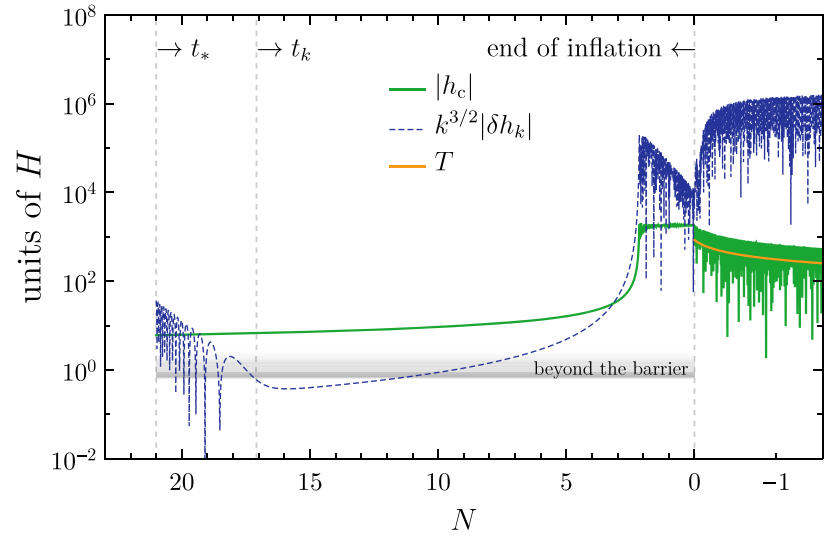

Fig. 2 Evolution of $h_{\mathrm{c}}$ and its perturbation $\delta h_{k}$ for the case $N_{*}>\bar{N}_{*}$ (or equivalently $h_{*}>\bar{h}_{*}$; see the text for details)

of motion for the Higgs background $h_{\mathrm{c}}$ and its perturbations $\delta h_{k}$, and compute the power spectrum $\mathscr{P}_{\zeta}$ of the comoving curvature perturbation $\zeta$.

The outcome is the following. Let us fix for the moment $h_{*}$, and denote by $\bar{N}_{*}$ the initial time which would give the right abundance of PBHs without the presence of $S$. By including $S$, the Higgs potential does not change for $h<m_{S}$, so that for $N_{*} \leq \bar{N}_{*}$ the evolution of the Higgs is not altered with respect to what was discussed in Ref. [1]: for $N_{*}<\bar{N}_{*}$, the final $\mathscr{P}_{\zeta}$ is too small to seed PBH. For $N_{*}>\bar{N}_{*}$, the Higgs field reaches its minimum at $m_{S}$ before the end of inflation, and starts oscillating around it. ${ }^{1}$ In the meantime, the tachyonic excitation of the Higgs fluctuations ceases, and $\delta h_{k}$ oscillates around zero with the same frequency as $h_{\mathrm{c}}$. The evolution for this case $N_{*}>\bar{N}_{*}$ is shown in Fig. 2 .

The redshift of $\delta h_{k}$ during this phase (in which the Higgs behaves as a matter fluid) slowly reduces the amplitude of $\zeta$ as $a^{-3 / 2}$, and $\mathscr{P}_{\zeta}$ decreases as $a^{-3}$ during the oscillation of $h_{\mathrm{c}}$ around its true minimum. ${ }^{2}$ The final value of $\mathscr{P}_{\zeta}$ for the mode $k_{*}=a\left(t_{*}\right) H$ (which exits the Hubble radius at $N_{*}$ ) is shown in Fig. 3.

Notice that the previous discussion proceeds in the same way if we fix a generic $N_{*}$ and identify what $\bar{h}_{*}$ leads to the right $\mathrm{PBH}$ abundance without the presence of $S$. For $h_{*} \leq \bar{h}_{*}$ the evolution is the same described in Ref. [1], whereas for $h_{*}>\bar{h}_{*}$ the curvature perturbation is slowly reduced at the end of inflation.

Figure 4 shows the final power spectrum $\mathscr{P}_{\zeta}$ as a function of the initial values $N_{*}$ and $h_{*}$. On the left-hand side of the

\footnotetext{
1 The Higgs mass in this true minimum is typically larger than $H$.

2 Both in Ref. [1] and in this paper we assume for simplicity a constant Hubble rate during inflation, in order not to specify an inflation model. This implies $\dot{\rho}_{\text {tot }}=\dot{\rho}_{h}$ during inflation. This approximation works less well during the oscillating phase in which the Higgs behaves as matter, and $\rho_{h} \sim a^{-3}$. In any case the qualitative behaviour of $\mathscr{P}_{\zeta}$ shown in Figs. 3 and 4 would be the same: the slope of $\mathscr{P}_{\zeta}$ for $N_{*}>\bar{N}_{*}$ in Fig. 3 would be steeper, without altering our conclusions.
}

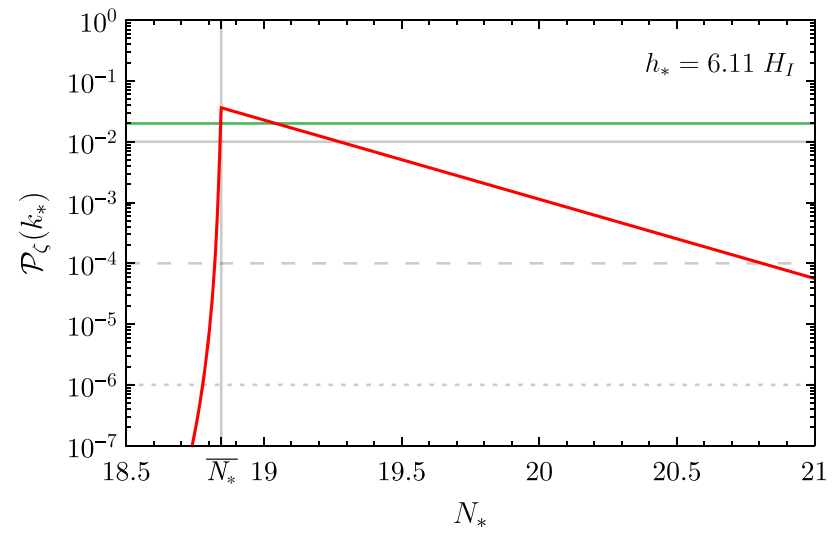

Fig. 3 Power spectrum $\mathscr{P}_{\zeta}$ as a function of the starting value $N_{*}$, for a fixed $h_{*}=6.11 \mathrm{H}$. The green line corresponds to a PBH abundance roughly of order unity, and the grey lines, yielding no PBHs, correspond to the cases shown in Fig. 4

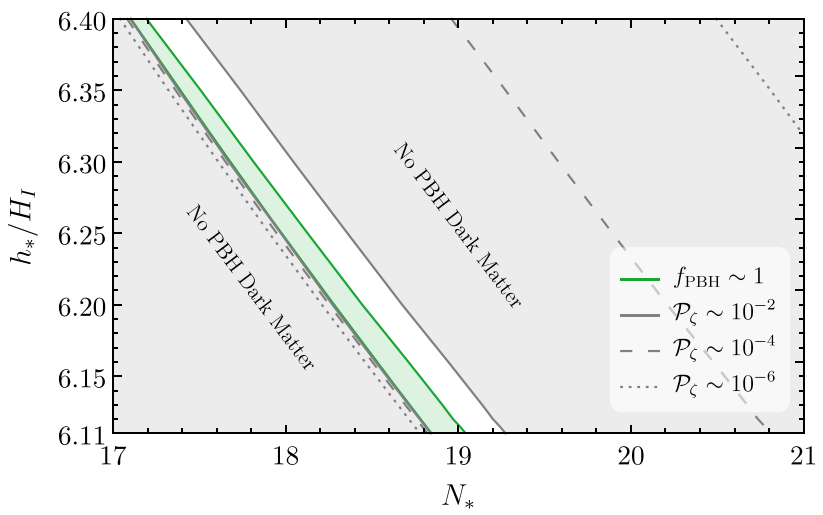

Fig. 4 Power spectrum $\mathscr{P}_{\zeta}$ as a function of the starting values $N_{*}, h_{*}$. The grey regions do not yield PBHs, and the green region corresponds to a non-negligible $\mathrm{PBH}$ abundance

plot we have the same situation as in Ref. [1], with a strong variation of the final $\mathscr{P}_{\zeta}$ even for per mille variations of $N_{*}$ and $h_{*}$. On the right-hand side of the plot we find the region which would have fallen into AdS without the threshold correction on $\lambda$ at $m_{S}$. If $\lambda$ is pushed to positive values at a scale close to $T_{\mathrm{RH}}$, then the Higgs is always rescued and there are no AdS regions which could form. Moreover, the dependence of $\mathscr{P}_{\zeta}$ on $N_{*}$ is much milder on the right side of Fig. 4, and a second region leading to the right PBH abundance is found.

For each initial value $h_{*}$, the spatial distribution of Higgs field values around $h_{*}$ after an $e$-fold follows a Gaussian distribution with standard deviation $H /(2 \pi)$. For each such value, Fig. 4 shows the corresponding final value for the power spectrum $\mathscr{P}_{\zeta}$, which in turn implies a PBH abundance. The total probability of getting a given overall $\mathrm{PBH}$ abundance in the universe is the convolution of these probabilities. We highlight all the region corresponding to $\mathscr{P}_{\zeta}\left(k_{*}\right) \sim$ $(0.02-0.04)$. Values larger than $\sim 0.02$ yield a too large PBH abundance. 
Nevertheless, these patches will be compensated by regions without $\mathrm{PBH}$ keeping the central value of the $\mathrm{PBH}$ abundance centered around the correct value. The corresponding inhomogeneities occur at very small scales and therefore do not constitute a problem.

Acknowledgements We thank A. Linde for discussions. A.R. and D.R. are supported by the Swiss National Science Foundation (SNSF), project Investigating the Nature of Dark Matter, project number: 200020-159223. The work of J.R.E. has been partly supported by the ERC Grant 669668-NEO-NAT-ERC-AdG-2014, the Spanish Ministry MINECO under Grants 2016-78022-P and FPA2014-55613-P, the Severo Ochoa excellence program of MINECO (Grants SEV-20160588 and SEV-2016-0597) and by the Generalitat de Catalunya Grant 2014-SGR-1450.

Open Access This article is distributed under the terms of the Creative Commons Attribution 4.0 International License (http://creativecomm ons.org/licenses/by/4.0/), which permits unrestricted use, distribution, and reproduction in any medium, provided you give appropriate credit to the original author(s) and the source, provide a link to the Creative Commons license, and indicate if changes were made. Funded by SCOAP ${ }^{3}$.

\section{References}

1. J.R. Espinosa, D. Racco, A. Riotto, A. Cosmological, Signature of the standard model Higgs vacuum instability: primordial black holes as dark matter. Phys. Rev. Lett. 120.12, 121301 (2018). arXiv:1710.11196 [hep-ph]

2. M. Sher, Electroweak Higgs potentials and vacuum stability. Phys. Rept. 179, 273-418 (1989)

3. P.B. Arnold, Can the electroweak vacuum be unstable? Phys. Rev. D40, 613 (1989)

4. G. Altarelli, G. Isidori, Lower limit on the Higgs mass in the standard model: an update. Phys. Lett. B 337, 141-144 (1994)

5. J.A. Casas, J.R. Espinosa, M. Quiros, Standard model stability bounds for new physics within LHC reach. Phys. Lett. B 382, 374382 (1996). arXiv:hep-ph/9603227 [hep-ph]

6. T. Hambye, K. Riesselmann, Matching conditions and Higgs mass upper bounds revisited. Phys. Rev. D 55, 7255-7262 (1997). arXiv:hep-ph/9610272 [hep-ph]
7. G. Isidori, G. Ridolfi, A. Strumia, On the metastability of the standard model vacuum. Nucl. Phys. B 609, 387-409 (2001). arXiv:hep-ph/0104016 [hep-ph]

8. J. Ellis, J.R. Espinosa, G.F. Giudice, A. Hoecker, A. Riotto, The probable fate of the standard model. Phys. Lett. B 679, 369-375 (2009). arXiv:0906.0954 [hep-ph]

9. F. Bezrukov, M. Yu. Kalmykov, B. A. Kniehl, M. Shaposhnikov, Higgs boson mass and new physics. In: JHEP 10 (2012). [275(2012)], p. 140. arXiv:1205.2893 [hep-ph]

10. A.V. Bednyakov, B.A. Kniehl, A.F. Pikelner, O.L. Veretin, Stability of the electroweak vacuum: gauge independence and advanced precision. Phys. Rev. Lett. 115.20, 201802 (2015). arXiv:1507.08833 [hep-ph]

11. J. Elias-Miro, J.R. Espinosa, G.F. Giudice, G. Isidori, A. Riotto, A. Strumia, Higgs mass implications on the stability of the electroweak vacuum. Phys. Lett. B 709, 222-228 (2012). arXiv:1112.3022 [hep-ph]

12. G. Degrassi, S. Di Vita, J. Elias-Miro, J. R. Espinosa, G. F. Giudice, G. Isidori, A. Strumia, Higgs mass and vacuum stability in the Standard Model at NNLO. In: JHEP 08 (2012), p. 098. arXiv: 1205.6497 [hep-ph]

13. D. Buttazzo, G. Degrassi, P.P. Giardino, G.F. Giudice, F. Sala, A. Salvio, A. Strumia, Investigating the near-criticality of the Higgs boson. JHEP 12, 089 (2013). arXiv:1307.3536 [hep-ph]

14. J.R. Espinosa, G.F. Giudice, A. Riotto, Cosmological implications of the Higgs mass measurement. JCAP 0805, 002 (2008). arXiv:0710.2484 [hep-ph]

15. C. Gross, A. Polosa, A. Strumia, A. Urbano, W. Xue, Dark matter in the standard model? In: (2018). arXiv:1803.10242 [hep-ph]

16. A.D. Linde, D.A. Linde, A. Mezhlumian, From the Big Bang theory to the theory of a stationary universe. Phys. Rev. D 49, 1783-1826 (1994). arXiv:gr-qc/9306035 [gr-qc]

17. A. Linde, V. Vanchurin, How many universes are in the multiverse? Phys. Rev. D 81, 083525 (2010). arXiv:0910.1589 [hep-th]

18. J. R. Espinosa, G. F. Giudice, E. Morgante, A. Riotto, L. Senatore, A. Strumia, N. Tetradis, The cosmological Higgstory of the vacuum instability. In: JHEP 09 (2015), p. 174. arXiv: 1505.04825 [hep-ph]

19. J. Elias-Miro, J. R. Espinosa, G. F. Giudice, H. M. Lee, A. Strumia, Stabilization of the electroweak vacuum by a scalar threshold effect. In: JHEP 06 (2012), p. 031. arXiv:1203.0237 [hep-ph] 